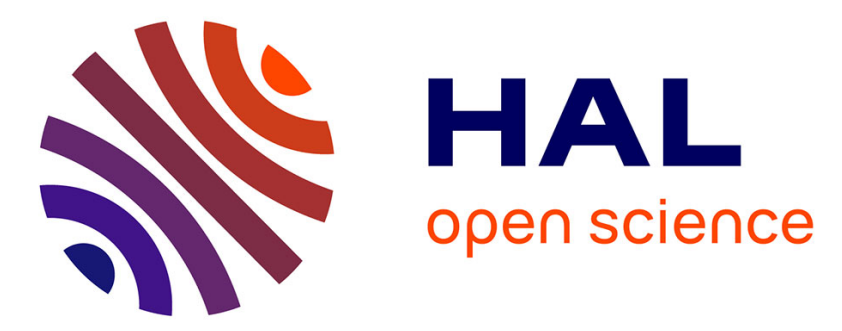

\title{
Non-Negative Spherical Deconvolution (NNSD) for Fiber Orientation Distribution Function Estimation
}

Jian Cheng, Rachid Deriche, Tianzi Jiang, Dinggang Shen, Pew-Thian Yap

\section{To cite this version:}

Jian Cheng, Rachid Deriche, Tianzi Jiang, Dinggang Shen, Pew-Thian Yap. Non-Negative Spherical Deconvolution (NNSD) for Fiber Orientation Distribution Function Estimation. MICCAI Workshop Computational Diffusion and Brain Connectivity, Sep 2013, Nagoya, Japan. pp.81-93, 10.1007/9783-319-02475-2_8. hal-00967829

\section{HAL Id: hal-00967829 https://hal.science/hal-00967829}

Submitted on 31 Mar 2014

HAL is a multi-disciplinary open access archive for the deposit and dissemination of scientific research documents, whether they are published or not. The documents may come from teaching and research institutions in France or abroad, or from public or private research centers.
L'archive ouverte pluridisciplinaire HAL, est destinée au dépôt et à la diffusion de documents scientifiques de niveau recherche, publiés ou non, émanant des établissements d'enseignement et de recherche français ou étrangers, des laboratoires publics ou privés. 


\title{
Non-Negative Spherical Deconvolution (NNSD) for Fiber Orientation Distribution Function Estimation
}

\author{
Jian Cheng, Rachid Deriche, Tianzi Jiang, Dinggang Shen, and Pew-Thian Yap
}

\begin{abstract}
In diffusion Magnetic Resonance Imaging (dMRI), Spherical Deconvolution (SD) is a commonly used approach for estimating the fiber Orientation Distribution Function (fODF). As a Probability Density Function (PDF) that characterizes the distribution of fiber orientations, the fODF is expected to be non-negative and to integrate to unity on the continuous unit sphere $\mathbb{S}^{2}$. However, many existing approaches, despite using continuous representation such as Spherical Harmonics (SH), impose non-negativity only on discretized points of $\mathbb{S}^{2}$. Therefore, nonnegativity is not guaranteed on the whole $\mathbb{S}^{2}$. Existing approaches are also known to exhibit false positive fODF peaks, especially in regions with low anisotropy, causing an over-estimation of the number of fascicles that traverse each voxel. This paper proposes a novel approach, called Non-Negative SD (NNSD), to overcome the above limitations. NNSD offers the following advantages. First, NNSD is the first $\mathrm{SH}$ based method that guarantees non-negativity of the fODF throughout the unit sphere. Second, unlike approaches such as Maximum Entropy SD (MESD), Cartesian Tensor Fiber Orientation Distribution (CT-FOD), and discrete representation based SD (DR-SD) techniques, the SH representation allows closed form of spherical integration, efficient computation in a low dimensional space resided by the SH coefficients, and accurate peak detection on the continuous domain defined by the unit sphere. Third, NNSD is significantly less susceptible to producing

Jian Cheng

University of North Carolina at Chapel Hill, e-mail: jian_cheng@med.unc.edu

Rachid Deriche

INRIA Sophia Antipolis, e-mail: Rachid.Derichedinria.fr

Tianzi Jiang

Institute of Automation, Chinese Academy of Sciences, e-mail: jiangtz@nlpr.ia.ac.cn

Dinggang Shen

University of North Carolina at Chapel Hill, e-mail: dgshen@med.unc.edu

Pew-Thian Yap

University of North Carolina at Chapel Hill, e-mail: ptyap@med.unc.edu
\end{abstract}


false positive peaks in regions with low anisotropy. Evaluations of NNSD in comparison with Constrained SD (CSD), MESD, and DR-SD (implemented using L1regularized least-squares with non-negative constraint), indicate that NNSD yields improved performance for both synthetic and real data. The performance gain is especially prominent for high resolution $(1.25 \mathrm{~mm})^{3}$ data.

\section{Introduction}

Diffusion MRI (dMRI) non-invasively reveals the microstructure of white matter by capturing the diffusion patterns of water molecules. The most widely used dMRI approach, Diffusion Tensor Imaging (DTI), is however incapable of describing complex diffusion processes due to its reliance on the assumption of Gaussian diffusion [10]. Hence, many methods have been developed in recent years to harness the power of High Angular Resolution Diffusion Imaging (HARDI) to characterize the non-Gaussian diffusion by evaluating quantities such as the Ensemble Average Propagator (EAP) [15, 5], the diffusion Orientation Distribution Function (dODF) [14, 7, 5], and the fiber Orientation Distribution Function (fODF) [13, 12, 1, 9].

A popular method, called Spherical Deconvolution (SD), has been shown to be effective for estimating the fODF by assuming that the measured diffusion-weighted signal can be obtained via spherically convolving a latent fODF with a fiber response function, which can be estimated from voxels known to be traversed by a single fascicle [13, 12, 9]. The fODF can hence be recovered via an inverse problem by deconvolving the signal with the fiber response function. SD methods can be classified into two categories, 1) continuous representation based SD (CR-SD), which is normally based on representation using Spherical Harmonics (SH) [13, 12, 1], and 2) discrete representation based SD (DR-SD), which is based on a discrete mixture of rotated versions of the fiber response function [9, 6, 11].

Existing SD methods in both categories have some common limitations. First, they often result in false-positive fODF peaks [13, 12, 1, 10, 9, 11, 17], especially in low anisotropic regions, such as the gray matter and cerebrospinal fluid (CSF) regions. Second, they normally fall short in ensuring that the estimated fODF is a proper probability density function, because non-negativity and unit integral over the unit sphere are not always enforced. Most SD methods, including the popular Constrained SD (CSD) [12], consider non-negativity only on discretized points of the unit sphere, but not the whole $\mathbb{S}^{2}[9,6,11]$. Thus, negative values are still a problem at points where non-negativity is not explicitly enforced. To our knowledge, Maximum Entropy SD (MESD) [1] and Cartesian Tensor Fiber Orientation Distribution (CT-FOD) [16, 17] are the only existing methods that ensure non-negativity throughout $\mathbb{S}^{2}$. However, these methods are inefficient without the closed form of spherical integration needed to compute the fODF and are susceptible to error associated with the numerical spherical integration. Ad-hoc normalization is also required in these methods to arrive at the fODF with unit integral. Third, for estimation 
of the fODF with reasonable accuracy, DR-SD methods require a significant number of rotated versions of the fiber response function in directions that are distributed densely on the unit sphere, significantly increasing the dimensionality and time cost of the optimization problem.

This paper proposes a novel CR-SD method, called Non-Negative Spherical Deconvolution (NNSD), for fODF estimation. Non-negativity is achieved by representing the square root of the fODF as a linear combination of SH basis functions. To our knowledge, NNSD is the first SH based SD method to guarantee non-negativity on the whole $\mathbb{S}^{2}$, not only on discretized points on the unit sphere, as in CSD [12]. Compared with MESD and CT-FOD, NNSD ensures unit integral and is more efficient due to the existence of a closed-form expression needed to arrive at the fODF. Moreover, NNSD significantly reduces the spurious peaks in regions with low anisotropy.

The rest of the paper is organized as follows. Section 2 provides an overview of three SD methods, i.e. CSD [12], MESD [1], and DR-SD (L1-regularized nonnegative least squares with non-negative constraint) [9, 11]. Section 3 describes NNSD and the associated Riemannian gradient descent algorithm. In Section 4. NNSD is empirically evaluated in comparison with the three methods discussed in Section 2

\section{Background on SD Methods}

If the fODF is represented as $\Phi(\mathbf{u})=\sum_{l=0}^{L} \sum_{m=-l}^{l} c_{l m} Y_{l}^{m}(\mathbf{u})$, and the axisymmetric fiber response function along the $z$-axis as

$$
H(\mathbf{u} \mid(0,0,1))=\sum_{l=0}^{L} h_{l} Y_{l}^{0}(\mathbf{u}),
$$

where $Y_{l}^{m}(\mathbf{u}), \mathbf{u} \in \mathbb{S}^{2}$, is the $l$ order and $m$ degree real Spherical Harmonic basis function [10, 7], then the diffusion signal can be represented as the convolution of the response function with the fODF [13]:

$$
E(\mathbf{u})=\int_{\mathbb{S}^{2}} H(\mathbf{u} \mid \mathbf{r}) \Phi(\mathbf{r}) \mathrm{d} \mathbf{r}=\sum_{l=0}^{L} \sum_{m=-l}^{l} \sqrt{\frac{4 \pi}{2 l+1}} c_{l m} h_{l} Y_{l}^{m}(\mathbf{u}) .
$$

Note that convolution here in conveniently performed via multiplication of the $\mathrm{SH}$ coefficients $c_{l m}$ and $h_{l}$. The SH coefficient vector $\boldsymbol{c}=\left(c_{00}, \ldots, c_{L L}\right)^{T}$ of the fODF can be estimated by minimizing

$$
\|\mathbf{M} \boldsymbol{c}-\mathbf{E}\|^{2},
$$

where $\mathbf{M}=\left[\sqrt{4 \pi / 2 l+1} h_{l} Y_{l}^{m}\left(\mathbf{u}_{i}\right)\right]$ is an $N$ by $(L+1)(L+2) / 2$ matrix, and $\mathbf{E}=$ $\left(E_{1}, \ldots, E_{N}\right)^{T}$ is a vector consisting of the signal measurements. The least squares solution is $\left(\mathbf{M}^{T} \mathbf{M}\right)^{-1} \mathbf{M}^{T} \mathbf{E}$. However, the least squares formulation does not take 
into account the non-negativity of the fODF. Constrained SD (CSD) [12] attempts to address this issue by iteratively suppressing the negative values by a discrete reconstruction on discrete points of the fODF, i.e., they solve

$$
\boldsymbol{c}^{(k+1)}=\underset{\boldsymbol{c}}{\arg \min }\|\mathbf{M} \boldsymbol{c}-\mathbf{E}\|^{2}+\lambda_{C S D}^{2}\left\|\mathbf{L}^{(k)} \boldsymbol{c}\right\|^{2},
$$

where $\mathbf{L}_{i, j}^{(k)}=P_{i, j}$ if $\left(\mathbf{P} c^{(k)}\right)_{i}<\tau, \mathbf{L}_{i, j}^{(k)}=0$ if $\left(\mathbf{P} c^{(k)}\right)_{i}>\tau$, and $\mathbf{P}$ is the SH matrix for reconstructing the fODF at discretized points on $\mathbb{S}^{2} . \tau$ is a threshold normally chosen as 0.1 [12]. Note that CSD imposes non-negativity only on a set of discretized points, not on the whole unit sphere. Although CSD significantly reduces the negative values compared with the original version of the SD technique described in [13], there is still a significant amount of negative values, even on points where non-negativity is imposed.

In MESD [1], a CR-SD method, a representation of the fODF is derived based on the maximum entropy principle:

$$
\Phi\left(\mathbf{r} \mid\left\{\lambda_{i}\right\}_{i=0}^{K}\right)=\exp \left(\lambda_{0}+\sum_{i=1}^{K} \lambda_{i} H\left(\boldsymbol{v}_{i} \mid \mathbf{r}\right)\right)
$$

which is an exponential function of mixture of fiber response functions determined by a set of parameters $\left\{\lambda_{i}\right\}_{i=0}^{K}$. These parameters can be determined by applying the Levenberg-Marquardt method to solve the following nonlinear least-squares problem:

$$
\min _{\left\{\lambda_{i}\right\}_{i=0}^{K}} \sum_{j=1}^{N}\left(\int_{\mathbb{S}^{2}} H\left(\mathbf{u}_{j} \mid \mathbf{r}\right) \Phi\left(\mathbf{r} \mid\left\{\lambda_{i}\right\}_{i=0}^{K}\right) \mathrm{d} \mathbf{r}-E_{j}\right)^{2} .
$$

The exponential representation naturally ensures non-negativity on $\mathbb{S}^{2}$. However, unlike (2), the spherical integration in (6) needs to be approximated numerically. An ad-hoc normalization of fODF is also needed to achieve unit integral after determining $\left\{\lambda_{i}\right\}_{i=0}^{K}$. Based on the method of Lagrange multipliers [1], the directions $\left\{\boldsymbol{v}_{i}\right\}_{i=1}^{K}$ should ideally be the $N$ sampling points corresponding to the signal measurements. However, to compromise between accuracy and speed, in practice $K<N$ evenly distributed points on $\mathbb{S}^{2}$ are used 1 .

In DR-SD, the fODF was represented using discretized points $\left\{\boldsymbol{w}_{i}=\Phi\left(\mathbf{u}_{i}\right)\right\}[9$, 6, 11]. The SD problem in this case is normally formulated as

$$
\min _{w}\|\mathbf{A} \boldsymbol{w}-\mathbf{E}\|^{2}+\lambda_{L 1}\|\boldsymbol{w}\|_{1}, \quad \text { s.t. } \boldsymbol{w} \geq 0,
$$

where $\mathbf{A}$ is the matrix with each column containing a rotated version of the fiber response function. For simplicity, we call this method the L1-regularized NonNegative Least Squares (L1NNLS). When $\lambda_{L 1}=0$, Eq. (7) becomes the NonNegative Least Squares (NNLS) [9, 6]. Since the fODF is represented using dis-

${ }^{1}$ Camino: http://cmic.cs.ucl.ac.uk/camino/ 
cretized points, its maxima are restricted to these points, not the continuous $\mathbb{S}^{2}$, eventually limiting the angular resolution for peak detection.

\section{Non-Negative Spherical Deconvolution (NNSD)}

The square root representation has been proposed for dODFs [3] and EAPs [4] and has been shown to be effective for non-negative estimation of these quantities [5]. In this work, we propose to employ this representation for non-negative estimation of the fODF. This is achieved by representing the square root of the fODF as a linear combination of SH basis functions:

$$
\Phi(\mathbf{u} \mid \boldsymbol{c})=\left(\sum_{l=0}^{L} \sum_{m=-l}^{l} c_{l m} Y_{l}^{m}(\mathbf{u})\right)^{2}=\sum_{\alpha=0}^{2 L} \sum_{\beta=-\alpha}^{\alpha}\left(\sum_{l, m}^{L} \sum_{l^{\prime}, m^{\prime}}^{L} c_{l m} c_{l^{\prime} m^{\prime}} Q_{l l^{\prime} \alpha}^{m m^{\prime} \beta}\right) Y_{\alpha}^{\beta}(\mathbf{u}),
$$

where $Q_{l l^{\prime} \alpha}^{m m^{\prime} \beta}=\int_{\mathbb{S}^{2}} Y_{l}^{m}(\mathbf{u}) Y_{l^{\prime}}^{m^{\prime}}(\mathbf{u}) Y_{\alpha}^{\beta}(\mathbf{u}) \mathrm{du}$ is a constant tensor computed by the integration of three sets of SH basis functions [5]. $Q_{l l^{\prime} \alpha}^{m m^{\prime} \beta}$ can be calculated from the Wigner 3-j symbol. Note that the sum over $\alpha$ is up to $2 L$, because if $\alpha>2 L$, then $Q_{l l^{\prime} \alpha}^{m m^{\prime} \beta}=0$ based on the property of Wigner 3-j symbol. In contrast to CSD, we use the SH basis function to represent the square root of the $f O D F$, instead of the fODF itself in CSD. Note that since $\int_{\mathbb{S}^{2}} \Phi(\mathbf{u}) \mathrm{d} \mathbf{u}=1$ and due to the orthogonality of the SH basis functions, we have $\int_{\mathbb{S}^{2}} \Phi(\mathbf{u}) \mathrm{d} \mathbf{u}=\|\boldsymbol{c}\|^{2}=1$. Based on Eq. [2), the convolved diffusion signal is

$$
E(\mathbf{u})=\sum_{\alpha=0}^{2 L} \sum_{\beta=-\alpha}^{\alpha} \sum_{l, m}^{L} \sum_{l^{\prime}, m^{\prime}}^{L} \sqrt{\frac{4 \pi}{2 \alpha+1}} c_{l m} c_{l^{\prime} m^{\prime}} Q_{l l^{\prime} \alpha}^{m m^{\prime} \beta} h_{\alpha} Y_{\alpha}^{\beta}(\mathbf{u})=\boldsymbol{c}^{T} \mathbf{K}(\mathbf{u}) \boldsymbol{c}
$$

where for any fixed $\mathbf{u}, \mathbf{K}(\mathbf{u})$ is a $(L+1) \times(L+2) / 2$ square matrix with the elements

$$
\mathbf{K}_{l l^{\prime}}^{m m^{\prime}}(\mathbf{u})=\sum_{\alpha=0}^{2 L} \sum_{\beta=-\alpha}^{\alpha} \sqrt{\frac{4 \pi}{2 \alpha+1}} Q_{l l^{\prime} \alpha}^{m m^{\prime} \beta} h_{\alpha} Y_{\alpha}^{\beta}(\mathbf{u})
$$

Based on Eq. 99, we propose to estimate $\boldsymbol{c}$ from the measured $\left\{E_{i}\right\}_{i=1}^{N}$ by minimizing

$$
J(\boldsymbol{c})=\frac{1}{2} \sum_{i=1}^{N}\left(\boldsymbol{c}^{T} \mathbf{K}(\mathbf{u}) \boldsymbol{c}-E_{i}\right)^{2}+\frac{1}{2} \boldsymbol{c}^{T} \Lambda \boldsymbol{c}, \quad \text { s.t. }\|\boldsymbol{c}\|=1,
$$

where $\Lambda$ is a diagonal matrix with elements $\Lambda_{l m}=\lambda_{N N S D} l^{2}(l+1)^{2}$ for the LaplaceBeltrami regularization [7]. The Riemannian gradient $\nabla J(\boldsymbol{c})$ is the projection of Euclidean gradient $\frac{\partial J(\boldsymbol{c})}{\partial \boldsymbol{c}}$ onto the tangent space of $\boldsymbol{c}$, i.e. 


$$
\nabla J(\boldsymbol{c})=\frac{\partial J(\boldsymbol{c})}{\partial \boldsymbol{c}}-\left(\boldsymbol{c}^{T} \frac{\partial J(\boldsymbol{c})}{\partial \boldsymbol{c}}\right) \boldsymbol{c}, \quad \frac{\partial J(\boldsymbol{c})}{\partial \boldsymbol{c}}=\sum_{i=1}^{N} 2\left(\boldsymbol{c}^{T} \mathbf{K}\left(\mathbf{u}_{i}\right) \boldsymbol{c}-E_{i}\right) \mathbf{K}\left(\mathbf{u}_{i}\right) \boldsymbol{c}+\Lambda \boldsymbol{c} .
$$

Then the Riemannian gradient descent of $J(\boldsymbol{c})$ is

$$
\boldsymbol{c}^{(k+1)}=\operatorname{Exp}_{\boldsymbol{c}^{(k)}}\left(-d t \frac{\nabla J(\boldsymbol{c})}{\|\nabla J(\boldsymbol{c})\|}\right), \quad \operatorname{Exp}_{\boldsymbol{c}}(\boldsymbol{v})=\boldsymbol{c} \cos \|\boldsymbol{v}\|+\frac{\boldsymbol{v}}{\|\boldsymbol{v}\|} \sin \|\boldsymbol{v}\|,
$$

where $\boldsymbol{c}^{(k)}$ is the estimated $\boldsymbol{c}$ in the $k$-th step, $d t$ is the step size chosen from the inexact line search in $\left(0, d t_{0}\right]$, and $\operatorname{Exp}_{\boldsymbol{c}}(\boldsymbol{v})$ is the exponential map on the sphere [3]. The whole process is summarized in Algorithm 1 . We use the isotropic fODF with $\boldsymbol{c}^{(0)}=(1,0, \ldots, 0)^{T}$ for initialization, and experimentally choose $d t_{0}=0.1$. We found that NNSD is robust to noise and $\lambda_{N N S D}=0$ works in most cases. To obtain a local minimum of $J(\boldsymbol{c})$, the standard stopping condition can be set as

$$
\frac{J\left(\boldsymbol{c}^{(k-1)}\right)-J\left(\boldsymbol{c}^{(k)}\right)}{J\left(\boldsymbol{c}^{(k-1)}\right)}<\delta .
$$

A small value for $\delta$ should be chosen such that NNSD converges to a local minimum of Eq. 111. Experimentally $\delta=10^{-2}$ works well in most cases. If $\delta$ is much smaller than $10^{-2}$, we found that although the cost function $J(\boldsymbol{c})$ becomes a little lower and the fODFs in regions with high anisotropy become a little shaper, the fODFs in regions with low anisotropy however become fuzzier with more spurious peaks. Thus we propose an adaptive stopping condition such that if $\operatorname{GFA}\left(\boldsymbol{c}^{(k)}\right)<T$ and $\frac{J\left(\boldsymbol{c}^{(k-1)}\right)-J\left(\boldsymbol{c}^{(k)}\right)}{J\left(\boldsymbol{c}^{(k-1)}\right)}<\delta_{0}$, or if $\operatorname{GFA}\left(\boldsymbol{c}^{(k)}\right) \geq T$ and $\frac{J\left(\boldsymbol{c}^{(k-1)}\right)-J\left(\boldsymbol{c}^{(k)}\right)}{J\left(\boldsymbol{c}^{(k-1)}\right)}<0.01 \delta_{0}$, then the gradient descent stops, where $\delta_{0}=10^{-2}, \operatorname{GFA}(\boldsymbol{c})=\sqrt{1-\frac{c_{00}^{2}}{\|\boldsymbol{c}\|^{2}}}=\sqrt{1-c_{00}^{2}}$ is the Generalized Fractional Anisotropy (GFA) [14], and $T \in[0,1]$ is a threshold depending on the noise level. For simplicity, we call NNSD with this adaptive stopping condition NNSD-ASC. When $T=0$, NNSD-ASC becomes NNSD with $\delta=0.01 \delta_{0}=10^{-4}$, and when $T=1$, NNSD-ASC becomes NNSD with $\delta=\delta_{0}=10^{-2}$. Both NNSD and NNSD-ASC converge fast, normally in a dozen of steps. Based on our C++ implementation, when $L=6$, fODFs in 1000 voxels can be estimated within 8 seconds using an ordinary laptop, which is much faster than MESD in Camino [1].

Note that in each iteration step, the fODF $\Phi\left(\mathbf{u} \mid \boldsymbol{c}^{(k)}\right)$ is naturally non-negative in the whole $\mathbb{S}^{2}$ due to the square operator in Eq. (8), and it has unit integral since $\left\|\boldsymbol{c}^{(k)}\right\|=1$.

\section{Experiments}

We compared the proposed NNSD and NNSD-ASC with CSD, MESD, and L1NNLS using synthetic and real data. 


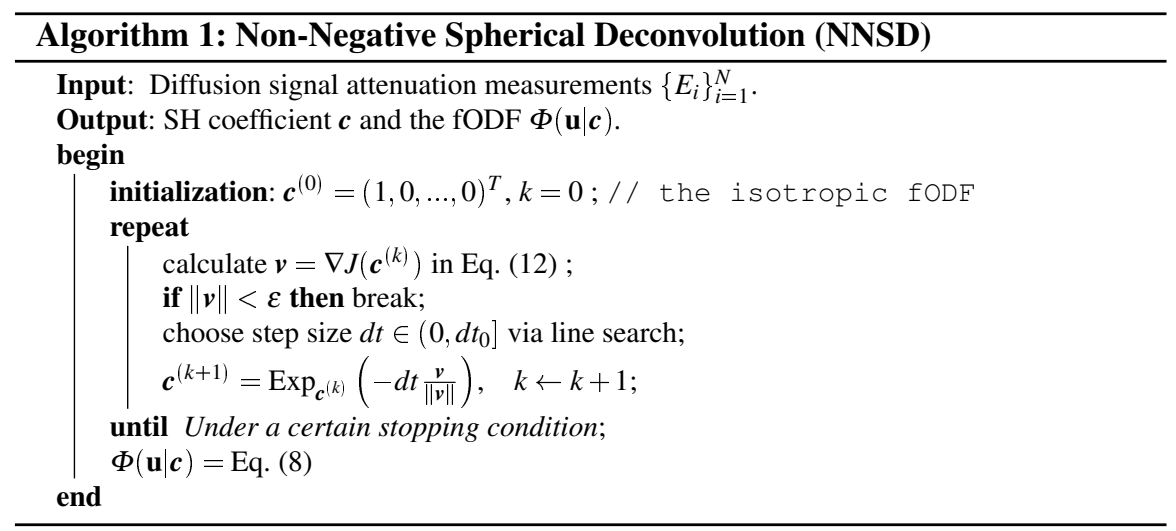

Parameters: Note that in all the following experiments, MRtrix ${ }^{2}$ with default parameters was used for CSD [12], and 321 evenly distributed orientations were used to generate $\mathbf{P}$ for CSD as suggested in [12, 9]. Accelerated MESD with $K=16$ was used as suggested in Camino. We experimentally set $\lambda_{L 1}=1$ for L1NNLS. Note that ideally the optimal $\lambda_{L 1}$ in Eq. (7) should be different in each voxel depending on $\mathbf{E}$. We normalize the columns of $\mathbf{A}$ and $\mathbf{E}$ to have unit norm so that we can use the same $\lambda_{L 1}$ in all cases. For fair comparison, we intentionally set $\lambda_{N N S D}=0$ to switch off Laplace-Beltrami regularization, and set $T=0.5$ in NNSD-ASC. For CSD, MESD, and NNSD, NNSD-ASC, the local maxima of the estimated fODF larger than the mean of the minimal and maximal values are detected by gradient ascent on the continuous unit sphere $\mathbb{S}^{2}$ [13, 12]. For L1NNLS, the peaks are detected from the 321 orientations.

Generation of Synthetic Data: Synthetic signals were generated using a mixture of tensor model $E(\mathbf{u})=\sum_{k=1}^{K} p_{k} \exp \left(-b \mathbf{u}^{T} \mathbf{D}_{k} \mathbf{u}\right)$ with $b=1500 \mathrm{~s} / \mathrm{mm}^{2}$ and 60 evenly distributed directions $\left\{\mathbf{u}_{i}\right\}$. For simulating crossings, two tensors with equal weights $p_{1}=p_{2}=0.5$ and common eigenvalues $\left(\lambda_{1}, \lambda_{2}, \lambda_{2}\right)$ but different orientations were used. Rician noise with signal-to-noise ratio (SNR) $1 / \sigma$ was added to the signal, where $\sigma$ is the standard deviation of the complex Gaussian noise.

Anisotropy and Non-Negativity: 1000 realizations of Rician noise corrupted signal were generated with $\mathrm{SNR}=15$ and 30 , respectively from a ground truth isotropic signal profile with eigenvalues $(0.7,0.7,0.7) \times 10^{-3} \mathrm{~mm}^{2} / \mathrm{s}$ and an anisotropic signal profile with eigenvalues $(1.7,0.2,0.2) \times 10^{-3} \mathrm{~mm}^{2} / \mathrm{s}$. The Gaussian fiber response function with eigenvalues $(1.7,0.2,0.2) \times 10^{-3} \mathrm{~mm}^{2} / \mathrm{s}$ was used in all methods. From the estimated fODFs, we recorded the GFA values [14] and the proportion of the negative fODF values. We ignored negative values close to zero and only take into consideration negative values with absolute values larger than $1 \%$ of the maximal fODF value. This evaluation was performed on 5121 points generated via tessellation of the unit sphere. The top left sub-figure in Fig. 1 1 shows that

\footnotetext{
${ }^{2}$ MRtrix: http://www.brain.org.au/software/mrtrix/
} 


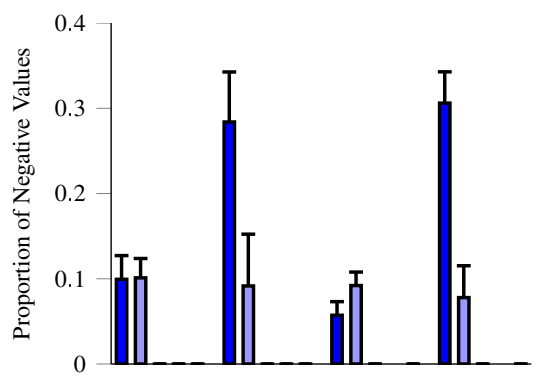

ISO, SNR=15 ANI, SNR=15 ISO, SNR=30 ANI, SNR=30

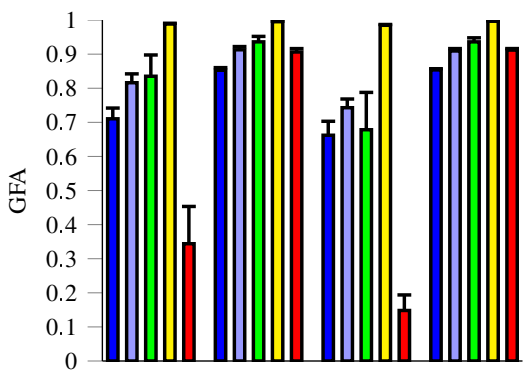

ISO, SNR=15 ANI, SNR=15 ISO, SNR=30 ANI, SNR=30

CSD, $L=6 \square$ CSD, $L=10 \square$ MESD $\square$ L1NNLS, $\lambda_{L 1}=1 \square$ NNSD-ASC, $L=6$

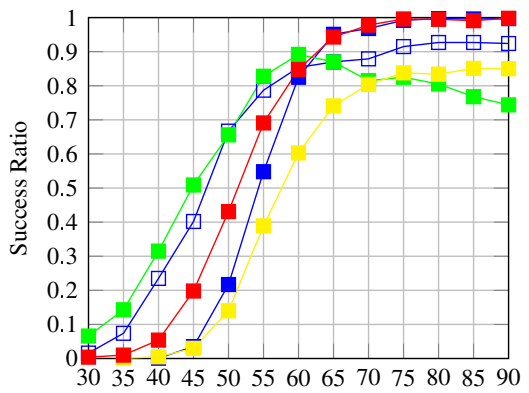

Crossing Angle (SNR=10)

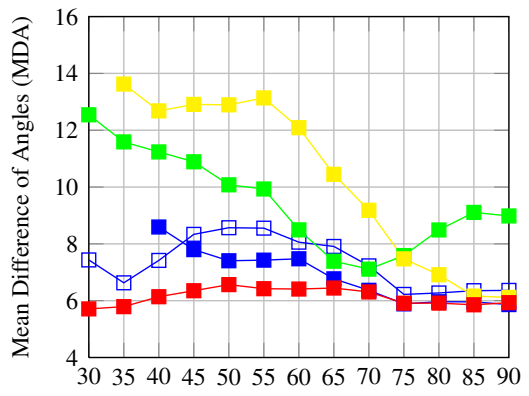

Crossing Angle $(\mathrm{SNR}=10)$

- $\mathrm{CSD}, L=6-$ 口- CSD , $L=10 \multimap$ MESD LINNLS - NNSD-ASC, $L=6$

Fig. 1 The first row shows the proportion of negative values and the GFA values of the fODFs estimated from the isotropic signal profiles (denoted as ISO) and anisotropic signal profiles (denoted as ANI). The error bars indicate the standard deviations. The second row shows the success ratios and the MDA values of the different methods for crossings generated with various angles.

although CSD considers non-negativity, it still suffers from negative values. NNSDASC and MESD guarantee non-negativity throughout $\mathbb{S}^{2}$. Note that the fODFs estimated by L1NNLS are non-negative on the discrete 321 points where non-negativity is imposed; however, since fODF value for other points are unknown, we cannot compute the proportion of negative values for L1NNLS. The top right sub-figure shows that CSD, MESD and L1NNLS obtain very high GFA even for isotropic diffusion, indicating an over-estimation of the number of fascicles. Only NNSD-ASC yields large contrast between isotropic and anisotropic signal profiles. Note that due to the sparsity constraint used in L1NNLS, the GFA values of fODFs estimated by L1NNLS for both isotropic and anisotropic signals are always close to 1 .

Simulation of Crossing Fibers: Corrupted signals were generated from two tensor model with SNR=10, eigenvalues $[1.7,0.2,0.2] \times 10^{-3} \mathrm{~mm}^{2} / \mathrm{s}$, and with different crossing angles in $\left[30^{\circ}, 90^{\circ}\right]$. For all methods, we estimated the fODFs with the same Gaussian fiber response and detected the local peaks. We recorded the success ratio, which is the proportion of trials with the correct number (i.e. two) of detected 
peaks, then calculated the Mean Difference of Angles (MDA) only in successful trials [7]. The second row in Fig. 1] shows the success ratio and the MDA values for different crossing angles. MESD and CSD with $L=10$ yield higher success ratios for small crossing angles, but lower success ratios for larger crossing angles. However, we are more concerned with large crossing angles, since this is more likely to happen in real data. With $L=6$, NNSD-ASC generally yields higher success ratios than CSD and the lowest MDA values among all methods. L1NNLS generally yields lower success ratios, probably because one has to tune $\lambda_{L 1}$ for different fiber configurations to achieve optimal results, while we had just simply set $\lambda_{L 1}=1$. L1NNLS yields larger MDA values than others, probably due to the limited angular resolution. The large MDA values given by MESD are probably due to a larger $K$ is needed for more accurate peak detection; this however requires a much longer computation time.

Real Data: Evaluation was also performed using real human data with $b=$ $2000 \mathrm{~s} / \mathrm{mm}^{2}, 120$ gradient directions, TR/TE $=12400 / 116 \mathrm{~ms}, 2 \mathrm{~mm}$ isotropic voxel dimensions. We set $L=6$ for CSD and NNSD, NNSD-ASC. MRtrix was used for CSD and for the estimation of the fiber response function from the voxels with FA $>0.7$ [13, 12]. The results are showed in Fig. 2 NNSD with $\delta=10^{-4}$ and NNSD-ASC with $T=0.5$ yield similar results, which means the outcome is insensitive to $T$ for this data. We calculated the GFA maps [14] from the estimated fODFs, and set them as the background color in every sub-figure. Consistent with the results in Fig. 11, the results in Fig. 2 for CSD, MESD and L1NNLS show a significant amount of false positive peaks, which is especially evident in the regions with low anisotropy, as indicated by the low background GFA values. Because of the sparsity consideration in L1NNLS, normally only less than 5 out of the 321 samples of fODFs have non-zero values, thus the fODFs by L1NNLS have very high GFA values close to 1, based on the GFA definition. NNSD and NNSD-ASC dramatically reduced the spurious peaks of the estimated fODFs; see the yellow circles in Fig. 2. Note that in isotropic regions, the fODFs estimated by NNSD and NNDSASC are very close to being isotropic. Although all fODFs estimated by NNSD have unit integral, the values of isotropic fODFs are around $\frac{1}{4 \pi}$ and the peaks of anisotropic fODFs are normally larger than 1 . Hence, when visualizing fODFs at the same scale, the isotropic fODFs estimated by NNSD/NNSD-ASC look like dots which have much small sizes compared with anisotropic fODFs. We would like to point out that most existing papers on SD overlaid the estimated fODF glyphs on a FA or GFA map calculated from other models like DTI, not from the estimated fODF itself [13, 12, 9, 11, 17]. The proposed NNSD and NNSD-ASC obtain a very clean fODF field with similar peaks as detected by CSD in highly anisotropic regions and more isotropic fODFs in the isotropic regions. 


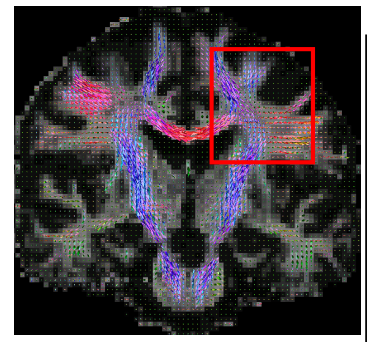

NNSD-ASC, $T=0.5, L=6$

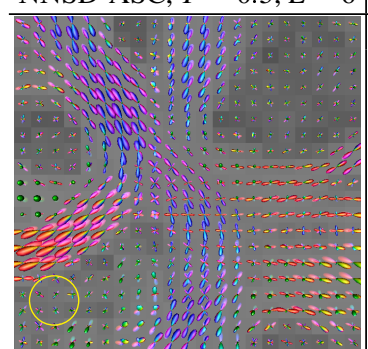

CSD, $L=6$, by MRtrix

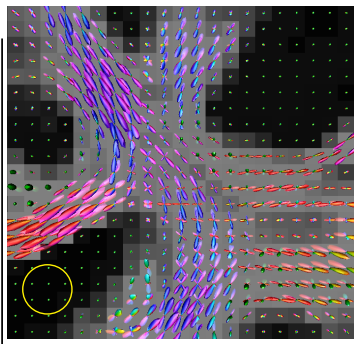

NNSD-ASC, $L=6, T=0.5$

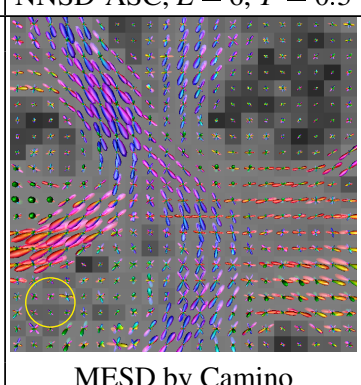

MESD by Camino

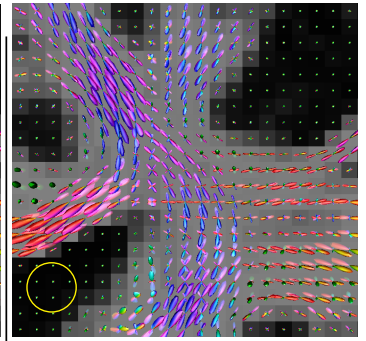

NNSD, $L=6, \delta=10^{-4}$

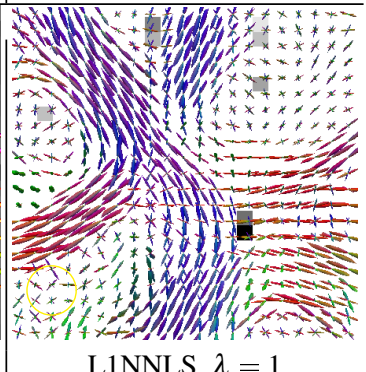

Fig. 2 First row: the whole coronal view of the fODF field using NNSD-ASC with $T=0.5$, and close-up views of results generated using NNSD-ASC and NNSD. Second row: close-up views for results generated using CSD, MESD, and L1NNLS. The colors of glyphs indicate directions. The backgrounds are the GFA maps.

\subsection{High-Resolution Data}

We also evaluated the SD methods using high resolution $(1.25 \mathrm{~mm})^{3}$ data obtained from the Human Connectome Project (HCP) ${ }^{3}$. The data is challenging because the SNR is low due to the small voxel dimensions. It has three shells, 90 directions per shell, $b=1000 / 2000 / 3000, \mathrm{~s} / \mathrm{mm}^{2}$. We performed NNSD $\left(\delta=10^{-4}\right)$, NNSDASC $\left(\delta=10^{-2}\right)$, CSD, MESD, L1NNLS on a single shell with $b=2000 \mathrm{~s} / \mathrm{mm}^{2}$; see Fig. 3. Although NNSD and NNSD-ASC yield results similar to the previous data (see Fig. 2), NNSD-ASC demonstrated its robustness to the spurious peaks in this high-resolution data. In the isotropic areas, only NNSD-ASC obtained isotropic fODFs, while other methods obtained many false positive peaks. See the yellow circles. In the anisotropic areas, NNSD and NNSD-ASC obtained the sharpest fODFs. fODFs by MESD are also very sharp in the anisotropic areas; however there are some small spurious lopes, as shown in the glyphs, similarly to the results shown in Fig. 2. fODFs estimated by CSD for this high-resolution data are not as sharp as those estimated by NNSD/NNSD-ASC and MESD. This can be observed in the areas marked by the yellow squares. L1NNLS as before obtained the most anisotropic GFA map, indicating spurious peaks in isotropic areas.

\footnotetext{
3 http://www.humanconnectome.org/data/
} 

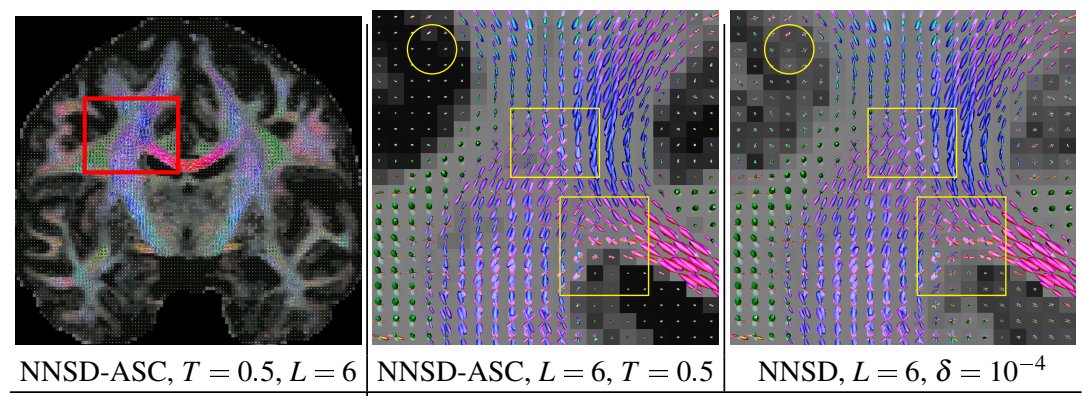

NNSD-ASC, $T=0.5, L=6$

NNSD-ASC, $L=6, T=0.5$

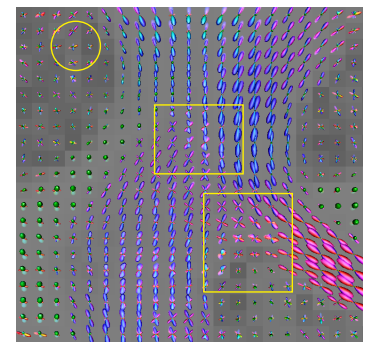

CSD, $L=6$, by MRtrix

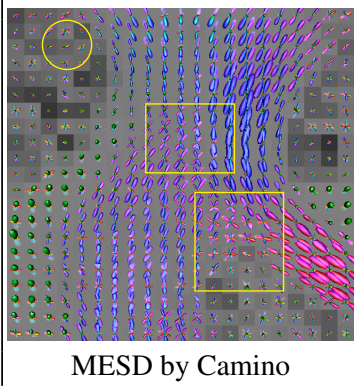

MESD by Camino

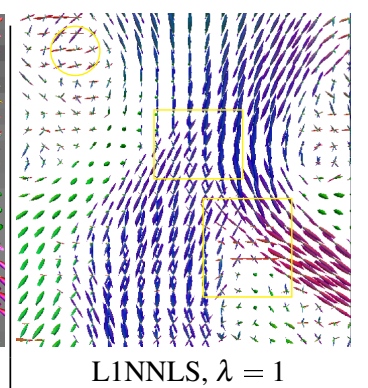

Fig. 3 First row: coronal view of the fODF field estimated using NNSD-ASC with $T=0.5$, and close-up views of results generated using NNSD-ASC and NNSD. Second row: close-up views of results generated using CSD, MESD and L1NNLS. The RGB color of glyphs indicates the directions. The backgrounds consist of the GFA maps.

\section{Discussion and Conclusion}

In all the above experiments, for fair comparisons between methods, we did not preprocess the data via denoising to enhance the SNR. We also did not consider spatia regularization for local smoothness. In practical applications, good denoising methods such as non-local means [8] and good spatial regularization can improve the final results. Although in this paper, we have only considered diffusion signals sampled from a single shell (e.g. one $b$ value), the proposed NNSD can be used in multiple shell data by considering a signal response function defined in the 3D space. The SH coefficients $\left\{h_{l}(b)\right\}$ for the different $b$-values can then be used in Eq. (1).

We participated ISBI 2013 HARDI reconstruction challeng $4^{4}$ using the proposed NNSD with non-local mean spatial regularization [2]. This challenge considered 3 categories of sampling schemes (DTI, HARDI, and multiple shell DSI-like schemes) and 3 SNRs ( $\mathrm{SNR}=10,20,30$ ), amounting to a total of 9 datasets. Compared with other methods in this challenge, NNSD was ranked as the best technique in terms of local fiber orientation accuracy in all 9 dataset 5

In this paper, we proposed a novel SD method, called Non-Negative SD (NNSD). NNSD is based on the square root representation of the fODF and can be solved by a

4 http://hardi.epfl.ch/static/events/2013_ISBI/

5 http://hardi.epfl.ch/static/events/2013_ISBI/_static/talk_Max.pdf 
Riemannian gradient descent algorithm with an adaptive stopping condition. Comparison with existing SD methods, including CSD, MESD, and L1NNLS, demonstrated the advantages of NNSD: 1) NNSD ensures that the estimated fODF is nonnegative with unit integral throughout $\mathbb{S}^{2} ; 2$ ) NNSD significantly reduces the false positive peaks and yields high contrast between isotropic and anisotropic regions; and 3) Due to the SH representation, NNSD is efficient and allows accurate peak detection on the continuous unit sphere. The experiments showed that the proposed NNSD-ASC works well in both isotropic and anisotropic areas, especially for the high-resolution data. The clean fODF field obtained by NNSD will be helpful for important applications such as fiber tractography.

\section{References}

1. Alexander, D.: Maximum entropy spherical deconvolution for diffusion MRI. In: Information Processing in Medical Imaging, pp. 27-57. Springer (2005)

2. Cheng, J., Deriche, R., Jiang, T., Shen, D., Yap, P.T.: Non-Local Non-Negative Spherical Deconvolution for Single and Multiple Shell Diffusion MRI. In: HARDI Reconstruction Challenge, International Symposium on Biomedical Imaging (ISBI) (2013)

3. Cheng, J., Ghosh, A., Jiang, T., Deriche, R.: A Riemannian Framework for Orientation Distribution Function Computing. In: Medical Image Computing and Computer-Assisted Intervention - MICCAI, vol. 5761, pp. 911-918 (2009)

4. Cheng, J., Ghosh, A., Jiang, T., Deriche, R.: Diffeomorphism Invariant Riemannian Framework for Ensemble Average Propagator Computing. In: Medical Image Computing and Computer-Assisted Intervention - MICCAI, LNCS, vol. 6892, pp. 98-106. Springer Berlin / Heidelberg (2011)

5. Cheng, J., Jiang, T., Deriche, R.: Nonnegative Definite EAP and ODF Estimation via a Unified Multi-Shell HARDI Reconstruction. In: Medical Image Computing and Computer-Assisted Intervention - MICCAI, LNCS, vol. 6892, pp. 98-106. Springer Berlin / Heidelberg (2012)

6. Dell'Acqua, F., Rizzo, G., Scifo, P., Clarke, R.A., Scotti, G., Fazio, F.: A model-based deconvolution approach to solve fiber crossing in diffusion-weighted MR imaging. Biomedical Engineering, IEEE Transactions on 54(3), 462-472 (2007)

7. Descoteaux, M., Angelino, E., Fitzgibbons, S., Deriche, R.: Regularized, Fast and Robust Analytical Q-ball Imaging. Magnetic Resonance in Medicine 58, 497-510 (2007)

8. Descoteaux, M., Wiest-Daessle, N., Prima, S., Barillot, C., Deriche, R.: Impact of Rician Adapted Non-local Means Filtering on HARDI. In: MICCAI (2008)

9. Jian, B., Vemuri, B.C.: A Unified Computational Framework for Deconvolution to Reconstruct Multiple Fibers from Diffusion Weighted MRI. IEEE Transactions on Medical Imaging 26, 1464-1471 (2007)

10. Johansen-Berg, H., Behrens, T.E.: Diffusion MRI: From quantitative measurement to In vivo neuroanatomy. Elsevier (2009)

11. Landman, B., Bogovic, J., Wan, H., El Zahraa, E., Bazin, P., Prince, J.: Resolution of crossing fibers with constrained compressed sensing using diffusion tensor mri. NeuroImage 59(3), $2175(2012)$

12. Tournier, J., Calamante, F., Connelly, A.: Robust determination of the fibre orientation distribution in diffusion MRI: non-negativity constrained super-resolved spherical deconvolution. NeuroImage 35(4), 1459-1472 (2007)

13. Tournier, J.D., Calamante, F., Gadian, D., Connelly, A.: direct estimation of the fiber orientation density function from diffusion-weighted MRI data using spherical deconvolution. NeuroImage 23, 1176-1185 (2004)

14. Tuch, D.S.: Q-ball imaging. Magnetic Resonance in Medicine 52, 1358-1372 (2004) 
15. Wedeen, V.J., Hagmann, P., Tseng, W.Y.I., Reese, T.G., Weisskoff, R.M.: Mapping Complex Tissue Architecture With Diffusion Spectrum Magnetic Resonance Imaging. Magnetic Resonance In Medicine 54, 1377-1386 (2005)

16. Weldeselassie, Y., Barmpoutis, A., Atkins, M.: Symmetric Positive-Definite Cartesian Tensor Orientation Distribution Functions (CT-ODF). In: Medical Image Computing and ComputerAssisted Intervention - MICCAI 2010 (2010)

17. Weldeselassie, Y.T., Barmpoutis, A., Stella Atkins, M.: Symmetric positive semi-definite cartesian tensor fiber orientation distributions (CT-FOD). Medical Image Analysis (2012) 



\section{Index}

Diffusion MRI,2

fiber Orientation Distribution Function, 2

Human Connectome Project, 10

Spherical Deconvolution, 2
L1-regularized Non-Negative Least Squares, 4

Constrained Spherical Deconvolution, 2 Maximum Entropy Spherical Deconvolution, 2

Non-Negative Spherical Deconvolution, 37 Spherical Harmonics, 2 